\title{
Simultaneous biodegradation of creosote-polycyclic aromatic hydrocarbons by a pyrene-degrading Mycobacterium
}

\author{
Zaira López • Joaquim Vila • José-Julio Ortega-Calvo • \\ Magdalena Grifoll
}

Published online: 1 February 2008

(C) Springer-Verlag 2008

\section{Erratum to: Appl Microbiol Biotechnol}

DOI 10.1007/s00253-007-1284-2

The original version of this article unfortunately contained a mistake. The presentation of Table 2 contains an error. The correct version is given below.

Table 2 Rates and extents of disappearance of creosote PAHs in cultures of Mycobacterium sp. AP1 ${ }^{\mathrm{a}}$

\begin{tabular}{|c|c|c|c|c|c|c|}
\hline \multirow[t]{2}{*}{ PAH } & \multicolumn{3}{|l|}{ Experimental } & \multicolumn{3}{|c|}{ Model predictions } \\
\hline & $C_{i}^{* \mathrm{~b}}(\mathrm{nmol} / \mathrm{ml})$ & $\begin{array}{l}\text { Max. rate } \\
\left(\text { pmol } \mathrm{ml}^{-1} \mathrm{~h}^{-1}\right)\end{array}$ & $\begin{array}{l}\text { Max. extent }{ }^{\mathrm{d}} \\
(\%)\end{array}$ & $\begin{array}{l}\text { Max. extent }{ }^{d} \\
(\%)\end{array}$ & $\begin{array}{l}F_{\text {slow }}{ }^{\mathrm{e}} \\
(\%)\end{array}$ & $\begin{array}{l}K_{\text {slow }}^{\mathrm{e}} \\
\left(\times 10^{-3} \mathrm{~h}^{-1}\right)\end{array}$ \\
\hline Fluorene & 2.96 & 57.2 & 38.2 & 40.4 & 98.5 & 0.70 \\
\hline Phenanthrene & 2.67 & 918.8 & 83.3 & 87.9 & 62.7 & 2.28 \\
\hline Anthracene & 1.16 & 150.6 & 36.9 & 37.6 & 77.1 & 0.29 \\
\hline Fluoranthene & 0.36 & 200.5 & 33.3 & 33.6 & 78.6 & 0.23 \\
\hline Pyrene & 0.20 & 47.1 & 11.5 & 10.7 & 92.6 & 0.05 \\
\hline Benzo $[a]$ anthracene & 0.08 & $16.0^{\mathrm{f}}$ & $7.3^{\mathrm{f}}$ & 7.6 & 100.0 & 0.12 \\
\hline Benzo $[a]$ pyrene & 0.03 & $10.3^{\mathrm{f}}$ & $6.0^{\mathrm{f}}$ & 6.3 & 100.0 & 0.11 \\
\hline
\end{tabular}

${ }^{a}$ Experimental values and model predictions were obtained from mean results of duplicate experiments

${ }^{\mathrm{b}} C_{i}^{*}$ Effective aqueous solubility calculated from molar fractions present at the beginning of the period of maximum biodegradation rate

${ }^{\mathrm{c}}$ Unless otherwise stated, maximum disappearance rates were calculated for the first $168 \mathrm{~h}$ of experimental period

${ }^{\mathrm{d}}$ Extent of compound loss at the end of experimental period $(720 \mathrm{~h})$

${ }^{\mathrm{e}} F_{\text {slow }}$ and $K_{\text {slow, }}$, pollutant fraction and first-order rate constant for slowly disappearing PAH, respectively

${ }^{\mathrm{f}}$ Maximum rates obtained after $168 \mathrm{~h}$

The online version of the original article can be found at http://dx.doi. org/10.1007/s00253-007-1284-2.

\section{Z. López $\cdot$ J. Vila $\cdot$ M. Grifoll $(\bowtie)$}

Departament de Microbiologia, Universitat de Barcelona,

Diagonal 645,

08028 Barcelona, Spain

e-mail: mgrifoll@ub.edu

\section{J.-J. Ortega-Calvo}

Instituto de Recursos Naturales y Agrobiología, C.S.I.C.,

Apartado 1052,

41080 Seville, Spain

Present address:

Z. López

Departamento Ciencias Médicas y de la Vida,

Centro Universitario de la Ciénega, Universidad de Guadalajara,

Ocotlán, Jalisco, Mexico 